\title{
单氟磷酸盐为深紫外非线性光学晶体探索提供新方向
}

\author{
梁飞，林哲帅* \\ 中国科学院理化技术研究所, 功能晶体与激光技术重点实验室, 北京 100190 \\ * 联系人, E-mail: zslin@mail.ipc.ac.cn
}

\section{Monofluorophosphates: A new candidate of deep-ultraviolet nonlinear optical materials}

\author{
Fei Liang \& Zheshuai Lin*
} Key Laboratory of Functional Crystals and Laser Technology, Technical Institute of Physics and Chemistry, Chinese Academy of Sciences, Beijing
100190, China

*Corresponding author, E-mail: zslin@mail.ipc.ac.cn

doi: 10.1360/N972018-01114

非线性光学晶体是一类具有重要战略价值的功能材 料, 可应用于红外军事对抗、激光频率转换、太赫兹光学、 超高分辨光刻、医疗诊断等 ${ }^{[1]}$. 其中, 深紫外非线性光学晶 体特指能够将激光频率转换为波长短于 $200 \mathrm{~nm}$ 的非线性 光学晶体. 自 20 世纪 80 年代起, 以陈创天院士为代表的我 国科学家开始了探索深紫外非线性光学材料的历程, 并发 现了氟硼铍酸钾 $\mathrm{KBe}_{2} \mathrm{BO}_{3} \mathrm{~F}_{2}(\mathrm{KBBF})$ 这一性能优良的新晶 体, 成功应用于 $N d$ : $Y A G$ 激光器的直接六倍频 $(177.3 \mathrm{~nm})$ 激光的稳定输出. 目前最高激光输出功率已经突破 $200 \mathrm{~mW}^{[2]}$, 处于国际领先地位. 2009年, Nature以“China's crystal cache" (中国的晶体秘藏) 为题, 介绍了 $\mathrm{KBBF}$ 晶体的 发展 ${ }^{[3]}$.

鉴于新型深紫外非线性光学材料体系的探索研究具 有重要的科学意义和实用价值, 近年来国内外多个课题组 积极进行探索, 方向涵盖了硼酸盐 ${ }^{[4]}$ 、碳酸盐 ${ }^{[5]}$ 、磷酸盐 ${ }^{[6]}$ 、 氟硼酸盐 ${ }^{[7]}$ 等, 取得了卓有成效的结果. 2018年10月19日, 北京师范大学吴立明课题组 ${ }^{[8]}$ 在 Chem. Mater. 发表题为 "Monofluorophosphates: A new source of deep-ultraviolet nonlinear optical materials” 的研究论文, 通过结构设计和 元素调控, 合成了 3 种具有优异非线性光学性能的单氟磷 酸盐化合物, 引起广泛关注.

吴立明课题组 ${ }^{[8]}$ 的出色工作是基于第一性原理理论计 算和实验表征两个方向完成的. 首先, 他们利用Gaussian和 VASP等量子化学软件包计算了 $\left(\mathrm{PO}_{4}\right)^{3-}$ 和 $\left(\mathrm{PO}_{3} \mathrm{~F}\right)^{2-}$ 基团的 能带特征(图 1(a)), 发现 $\left(\mathrm{PO}_{3} \mathrm{~F}\right)^{2-}$ 基团拥有比 $\left(\mathrm{PO}_{4}\right)^{3-}$ 更宽的 带隙、更强的极化各向异性和更高的一阶超极化率. $\left(\mathrm{PO}_{3} \mathrm{~F}\right)^{2-}$ 基团的分子带隙高达9.4 eV, 紫外吸收截止边预
计在 $132 \mathrm{~nm}$, 非常适合作为深紫外非线性光学材料的构筑 集团. 然后, 他们在无机晶体数据库中笁选了约 100 种单 氟磷酸盐化合物, 综合考虑晶体结构和元素组成, 选择了 5 种可能的单氟磷酸盐化合物作为备选材料. 采用第一性 原理方法计算了它们的电子结构及非线性光学性质, 最终 选定 $\left(\mathrm{NH}_{4}\right)_{2} \mathrm{PO}_{3} \mathrm{~F}, \quad\left[\mathrm{C}\left(\mathrm{NH}_{2}\right)_{3}\right]_{2} \mathrm{PO}_{3} \mathrm{~F}$ 和 $\mathrm{NaNH}_{4} \mathrm{PO}_{3} \mathrm{~F} \cdot \mathrm{H}_{2} \mathrm{O}$ 三种 化合物作为深紫外非线性光学晶体的备选材料.

随后, 吴立明课题组采用了多样的化学合成方法(固 相反应、离子交换、溶液沉淀)合成了这 3 种材料并重新解 析了晶体结构. 紫外可见漫反射光谱表明, 3 种化合物的紫 外透过截止边均在 $200 \mathrm{~nm}$ 以下, 且表现出较强的非线性光 学响应, 其中 $\mathrm{NaNH}_{4} \mathrm{PO}_{3} \mathrm{~F} \cdot \mathrm{H}_{2} \mathrm{O}$ 的综合性能最优. 它属于单 斜 $P \mathrm{c}$ 空间群(图1(b)), 紫外截止边是176 nm(图1(c)), 1064 $\mathrm{nm}$ 的倍频信号是 $\mathrm{KH}_{2} \mathrm{PO}_{4}(\mathrm{KDP})$ 晶体的 1.1倍(图 1(d)), 532 $\mathrm{nm}$ 的倍频信号是 $\beta-\mathrm{BaB}_{2} \mathrm{O}_{4}(\mathrm{BBO})$ 晶体的 1/3(图 1(e)), 并且 都可以实现相位匹配. 初步测量的抗激光损伤阈值为 $118.1 \mathrm{MW} / \mathrm{cm}^{2}$, 是同等条件下 KDP晶体的 3 倍. 理论计算 的最大非线性光学系数分量为 $0.72 \mathrm{pm} / \mathrm{V}$, 与 $\mathrm{KBBF}$ 晶体 $\left(d_{11}=0.49 \mathrm{pm} / \mathrm{V}\right)$ 相当. 初步的晶体生长实验已经得到了尺 寸为 $14 \mathrm{~mm} \times 9 \mathrm{~mm} \times 2.3 \mathrm{~mm}$ 的单晶(图1(f)), 未来有望获得 尺寸更大、质量更高的晶体.

单氟磷酸盐作为一个古老的材料体系, 最早可以追溯 到 20 世纪初. 尽管已经被广泛地应用于牙膏、口香糖、食品 添加剂等工业领域, 但是它们的非线性光学性能从未得到 关注. 吴立明课题组这一工作的意义在于展现了单氟磷酸 盐在非线性光学材料领域的应用潜力, 扩展了其潜在的应 用范围. 结合理论计算和实验表征, 预测并证实了 3 种单 
(a)

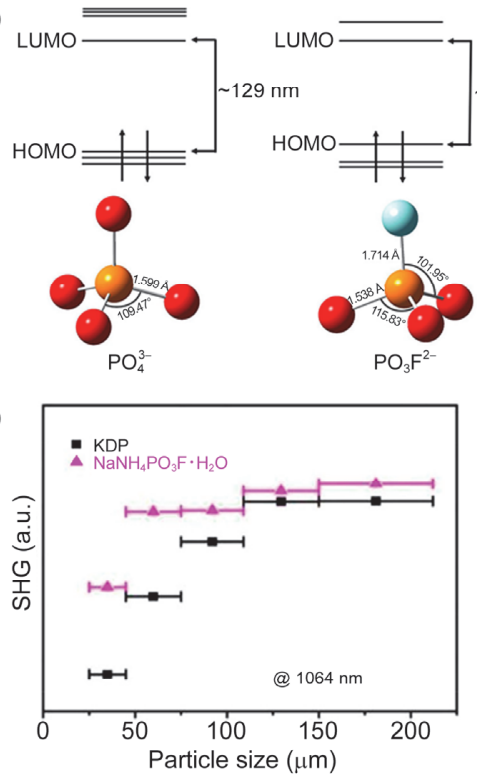

(b)

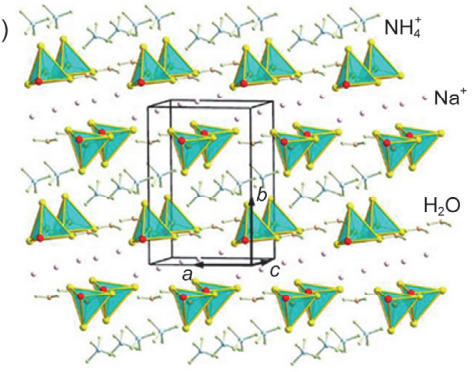

(e)

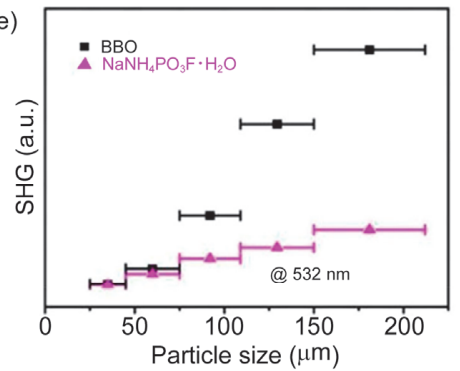

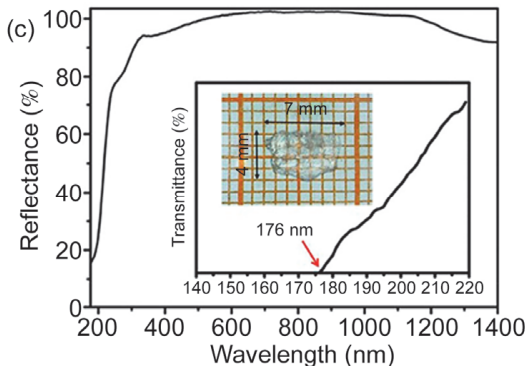

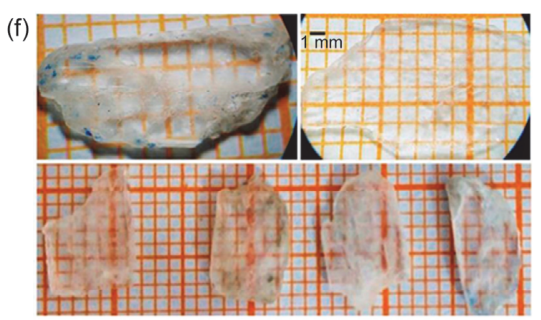

图 1 (网络版彩色)(a) 理论计算的 $\left(\mathrm{PO}_{4}\right)^{3-}$ 和 $\left(\mathrm{PO}_{3} \mathrm{~F}\right)^{2-}$ 基团的最高占据轨道和最低未占据轨道; (b) $\mathrm{NaNH}_{4} \mathrm{PO}_{3} \mathrm{~F} \cdot \mathrm{H}_{2} \mathrm{O}$ 的晶体结构; (c) 深紫外透过 光谱; (d) $1064 \mathrm{~nm}$ 的倍频信号; (e) $532 \mathrm{~nm}$ 的倍频信号; (f) $\mathrm{NaNH}_{4} \mathrm{PO}_{3} \mathrm{~F} \cdot \mathrm{H}_{2} \mathrm{O}$ 的晶体照片 ${ }^{[10]}$

Figure 1 (Color online) (a) The calculated highest occupied molecular orbitals (HOMO) and lowest unoccupied molecular orbitals (LUMO) of $\left(\mathrm{PO}_{4}\right)^{3-}$ and $\left(\mathrm{PO}_{3} \mathrm{~F}\right)^{2-}$; (b) crystal structure, (c) transmittance spectrum, (d) powder double frequency signal under 1064 nm, (e) powder double frequency signal under $532 \mathrm{~nm}$, and (f) photograph of $\mathrm{NaNH}_{4} \mathrm{PO}_{3} \mathrm{~F} \cdot \mathrm{H}_{2} \mathrm{O}$

氟磷酸盐化合物的倍频效应和相位匹配能力，突破了 $\left(\mathrm{PO}_{4}\right)^{3-}$ 基团中单一配位元素的局限, 从而极大丰富了深紫 外非线性光学材料的探索方向, 意义十分重大.

当然, 我们也不应忽视, 目前这一体系的晶体质量还 有待提高, 其深紫外谐波输出能力还有待实验证实, 因此 距离实际应用还有差距. 而通过理论计算、实验合成、晶
体生长、性能表征这一完整的科学研究路线, 揭示晶体结 构和物理性能之间的关系, 发现新型的光学功能材料, 还 大有挖掘空间. 随着高通量科学计算和实验合成能力的提 升, 我们相信会有更多的类似体系涌现. 吴立明课题组的 工作就是一个理论计算结合实验合成探索新型功能材料 的典型范例.

\section{参考文献}

1 Garmire E. Nonlinear optics in daily life. Opt Express, 2013, 21: 30532-30544

$2 \mathrm{Xu} \mathrm{B}$, Liu L, Wang X, et al. Generation of high power $200 \mathrm{~mW}$ laser radiation at $177.3 \mathrm{~nm}$ in $\mathrm{KBe}_{2} \mathrm{BO}_{3} \mathrm{~F}_{2}$ crystal. Appl Phys B Lasers Opt, 2015, 121: 489-494 Cyranoski D. Materials science: China's crystal cache. Nature, 2009, 457: 953-955

4 Zhao S, Gong P, Bai L, et al. Beryllium-free $\mathrm{Li}_{4} \mathrm{Sr}\left(\mathrm{BO}_{3}\right)_{2}$ for deep-ultraviolet nonlinear optical applications. Nat Commun, $2014,5: 4019$

5 Zou G, Ye N, Huang L, et al. Alkaline-alkaline earth fluoride carbonate crystals $\mathrm{ABCO}_{3} \mathrm{~F}(\mathrm{~A}=\mathrm{K}, \mathrm{Rb}, \mathrm{Cs}$; $\mathrm{B}=\mathrm{Ca}, \mathrm{Sr}, \mathrm{Ba})$ as nonlinear optical materials. J Am Chem Soc, 2011, 133: 20001-20007

6 Yu P, Wu L M, Zhou L J, et al. Deep-ultraviolet nonlinear optical crystals: $\mathrm{Ba}_{3} \mathrm{P}_{3} \mathrm{O}_{10} \mathrm{X}$ (X=Cl, Br). J Am Chem Soc, 2014, 136: 480-487

7 Shi G, Wang Y, Zhang F, et al. Finding the next deep-ultraviolet nonlinear optical material: $\mathrm{NH}_{4} \mathrm{~B}_{4} \mathrm{O}_{6} \mathrm{~F}$. J Am Chem Soc, 2017, 139: 10645-10648

8 Xiong L, Chen J, Lu J, et al. Monofluorophosphates: A new source of deep-ultraviolet nonlinear optical materials. Chem Mater, 2018, doi: 10.1021/acs.chemmater.8b03310 